\title{
VARIAÇÃO DAS PROPRIEDADES QUÍMICAS E REOLÓGICAS DA XANTANA PRUNI MEDIANTE ALTERAÇÕES DOS PARÂMETROS OPERACIONAIS AGITAÇÃO E AERAÇÃO
}

\author{
F. G. A. GAUTÉRIO ${ }^{1}$, A. C. R. MILCZARSKI ${ }^{2}$, C. A. BALBINOT-FILHO ${ }^{1}$, P. DIAZ ${ }^{2}$, A. da S. \\ MOREIRA $^{2}$ e C. T. VENDRUSCOLO ${ }^{2}$ \\ ${ }^{1}$ Universidade Federal do Pampa, Bagé, RS, Engenharia de Alimentos \\ ${ }^{2}$ Universidade Federal de Pelotas, Pelotas, RS, Centro de Desenvolvimento Tecnológico \\ E-mail para contato: fernandagauterio@unipampa.edu.br
}

\begin{abstract}
RESUMO - Objetivou-se obter um agente viscosificante de elevada performance para aplicação em fluidos de perfuração poços de petróleo. Para isto, produziu-se xantana a partir do cultivo de $X$. arboricola pv pruni em biorreator de $5 \mathrm{~L}$, mediante condições de agitação e aeração diferenciadas conforme patente WO2006047845. O biopolímero foi caracterizado quanto à viscosidade viscoelasticidade e aos parâmetros reológicos índice de consistência e índice de fluxo pelo ajuste do modelo de Ostwald-de-Waelle; teores de piruvato e acetil, e análise qualitativa dos monossacarídeos. As variações dos parâmetros proporcionaram atingir condições desejadas à produção d como viscosidade elevada e baixo conteúdo de piruvato; características estas necessárias, respectivamente, para aplicação em fluido de perfuração e recuperação terciária de petróleo.
\end{abstract}

\section{INTRODUÇÃO}

A investigação de natureza reológica, como a determinação da viscosidade e do comportamento viscoelástico e força gel do viscosificante a ser utilizado, são fundamentais para uma melhor compreensão e previsão para a capacidade de sustentação de sólidos gerados na perfuração de poços de petróleo e na recuperação terciária de poços maduros. A formulação correta dos fluidos de perfuração é um dos pontos críticos do processo, já que estes são misturas complexas e inadequabilidade na formulação ou qualidade de um ou mais constituintes pode provocar desde erosão e inchamento até desmoronamento do poço perfurado. Pode ocorrer também encerramento da broca ou aprisionamento da coluna de perfuração, com significativos prejuízos econômicos.

Xantana é um bioproduto polissacarídico produzido por bactérias do gênero Xanthomonas, com amplo interesse às indústrias petrolíferas, alimentícias, farmacêuticas, e outras. Todas as xantanas apresentam capacidade de formarem soluções viscosas e, algumas, como a xantana pruni, produto desenvolvido com tecnologia genuinamente nacional e já patenteado, formam géis hidrossolúveis, mesmo em baixas concentrações, que lhe fornecem propriedades reológicas únicas (Borges et al., 2009; Oliveira et al., 2012), sendo capaz de modificar profundamente o comportamento de escoamento das soluções que a contém. A xantana, apesar de possuir custo 


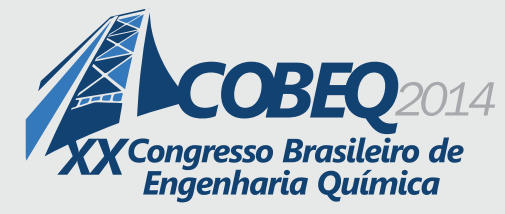

19 a 22 de outubro de 2014

Florianópolis/SC

mais elevado que os polímeros utilizados tradicionalmente é o único bioproduto conhecido, até o momento, capaz de apresentar, simultaneamente, alta viscosidade, mesmo em baixas concentrações, pseudoplasticidade, uma razoável tixotropia, resistência térmica a, no mínimo, $65^{\circ} \mathrm{C}$ e elevada compatibilidade com sais mono e divalentes, o que lhe confere a versatilidade de poder ser utilizada tanto em fluidos utilizando água doce como salgada. O fluido à base de água, como o que utiliza xantana, é considerado um dos mais econômicos, em função do excelente desempenho; tem ainda outras vantagens: não sofre combustão, é de fácil utilização e considerado ecologicamente correto; este último por ser a xantana um bio-produto biodegradável, além de biocompatível com o ambiente de exploração de petróleo, podendo ser considerada ecologicamente segura, pois é atóxica e não sofre bioacumulação, requisitos fundamentais para preservação do meio ambiente (Farias et a.,l 2009).

A xantana quando em solução possui comportamento não newtoniano (viscosidade é alterada com a elevação da taxa de cisalhamento) sendo, também, pseudoplástica, pois sua viscosidade diminui conforme o aumento da taxa de cisalhamento, recuperando a viscosidade, em maior ou menor grau, rapidamente quando a tensão de cisalhamento é removida. Quando em repouso ou em baixas taxas de cisalhamento, as associações das cadeias de xantana encontram-se estabilizadas por ligações de hidrogênio. Quando a xantana é submetida ao cisalhamento, a extensão da agregação é reduzida pelo alinhamento das cadeias, resultando em uma diminuição da viscosidade do polímero (Xuewu et al., 1996). As propriedades químicas e reológicas podem variar em função do microrganismo, parâmetros utilizados na produção (Torres et al., 1993).

Alterações nas propriedades da xantana são passíveis de serem obtidas por quem domina a técnica de produção e dispõe de cepas adequadas (Borges et al., 2009); dessa forma, o processo pode ser direcionado para se obter o produto com características específicas desejadas. Pelo exposto acima, o trabalho objetivou caracterizar a xantana pruni quanto às propriedades químicas e reológicas, mediante alterações de agitação e aeração.

\section{MATERIAL E MÉTODOS}

\subsection{Multiplicação celular e condução dos processos fermentativos}

$X$. arboricola pv pruni EDE foi empregada para a produção do inóculo em incubador agitador rotativo (modelo B. Braun Biotech International®) durante $24 \mathrm{~h}$, conforme patente WO/2006047845 (Universidade Federal de Pelotas, 2006). As fermentações foram do tipo submersa, mediante condições diferenciadas de agitação e aeração (Tabela 1), perfazendo 9 tratamentos (WO2006047845), em fermentador (modelo Biostat B de 5L, B. Braun Biotech International $\left.{ }^{\circledR}\right)$ de $5 \mathrm{~L}$ por $72 \mathrm{~h}$. $\mathrm{O} \mathrm{pH}$ foi ajustado inicialmente em 7,0 e não mais controlado durante o processo.

\subsection{Recuperação e caracterização das xantanas produzidas}

Recuperação das xantanas: As xantanas foram recuperadas, mediante insolubilização pela adição de etanol $96^{\circ} \mathrm{GL}$ (Vendruscolo et al., 2000). Xantanas comerciais de grau alimentício 


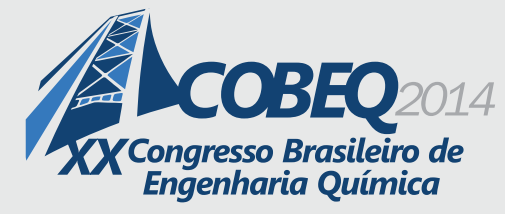

(Fufeng®) e específica para indústria petrolífera (Kelco®) foram utilizadas como controle.

Caracterização reológica: Soluções de xantana $1 \%(\mathrm{~m} / \mathrm{v})$ foram preparadas (Burdock, 1997) para determinação das curvas de viscosidade (modo rotativo) e viscoelasticidade (modo oscilatório) em reômetro (RheoStress modelo RS150), a $25^{\circ} \mathrm{C}$. Os parâmetros reológicos índice de consistência (K) e índice de fluxo (n) foram obtidos pelo ajuste do modelo Ostwald-de-Waelle.

Caracterização química: Os teores de acetil e piruvato foram mensurados de acordo com McComb e McCready (1957), Sloneker e Orentas (1962), respectivamente, e submetidos à Análise de Variância e Teste de Tukey com $\alpha$ de 5\%. A determinação qualitativa dos monossacarídeos foi conduzida conforme Moreira et al. (1998, modificado), empregando como padrões glicose, ramnose, manose e ácido glicurônico.

Tabela 1 - Combinações de agitação e aeração nos diferentes tratamentos para produção de xantana

\begin{tabular}{c|c|c}
\hline Tratamentos & Agitação* & Aeração* $^{*}$ \\
\hline 1 & Baixa & baixa \\
2 & Elevada & baixa \\
3 & elevada \\
4 & Elevada & elevada \\
5 & mais baixa & intermediária \\
6 & mais elevada & intermediária \\
7 & Intermediária & mais baixa \\
8 & Intermediária & mais elevada \\
9 & Intermediária & intermediária \\
\hline
\end{tabular}

\section{RESULTADOS E DISCUSSÃO}

As condições operacionais influíram na viscosidade das soluções das xantanas produzidas. Observou-se que todas as xantanas pruni apresentaram comportamento não newtoniano e pseudoplástico (Figura 1), como esperado (Xuewu et al. 1996). A viscosidade da xantana obtida no tratamento 7 foi a maior, incluindo as amostras comerciais; as obtidas nos demais tratamentos foram menos viscosas que as comerciais. Analisando a Figura 1 observa-se que a viscosidade foi reduzida pelo emprego de valores mais baixos (5) e elevados (8) de agitação e aeração, respectivamente. Por outro lado, a agitação intermediária combinada com aerações muito baixas (7) ou intermediárias (9) beneficiou a viscosidade do polímero. Em agitação intermediária, a mais baixa aeração influenciou positivamente a viscosidade (7); por outro lado, o polímero teve sua viscosidade influenciada negativamente pela mais elevada aeração (8). Pode-se, a partir destes

\footnotetext{
${ }^{1}$ As condições operacionais de agitação e aeração utilizadas nos experimentos estão protegidas por patente WO2006047845 (Vendruscolo, C. T; Vendruscolo, J. L. S; Moreira, A. S. (2006). Processo de produção de biopolímero tipo xantana, biopolímero obtido, seus usos; meio de cultura para crescimento de Xanthomonas e uso da mesma para produção de biopolímero. World Intellectual Property Organization (WO2006047845)). Devido ao fato desta patente estar em fase de comercialização, o Departamento Jurídico da Universidade Federal de Pelotas-UFPel não nos permite divulgação destes valores.
} 


\section{COBEQ 2014 Engenharia Química}

resultados, inferir-se que a aeração exerceu maior influência sobre a viscosidade; a elevada agitação não foi suficiente para compensar a baixa aeração (2). Quando empregados valores extremos de aeração foram verificadas viscosidades intermediárias e semelhantes entre si (1 a 6).

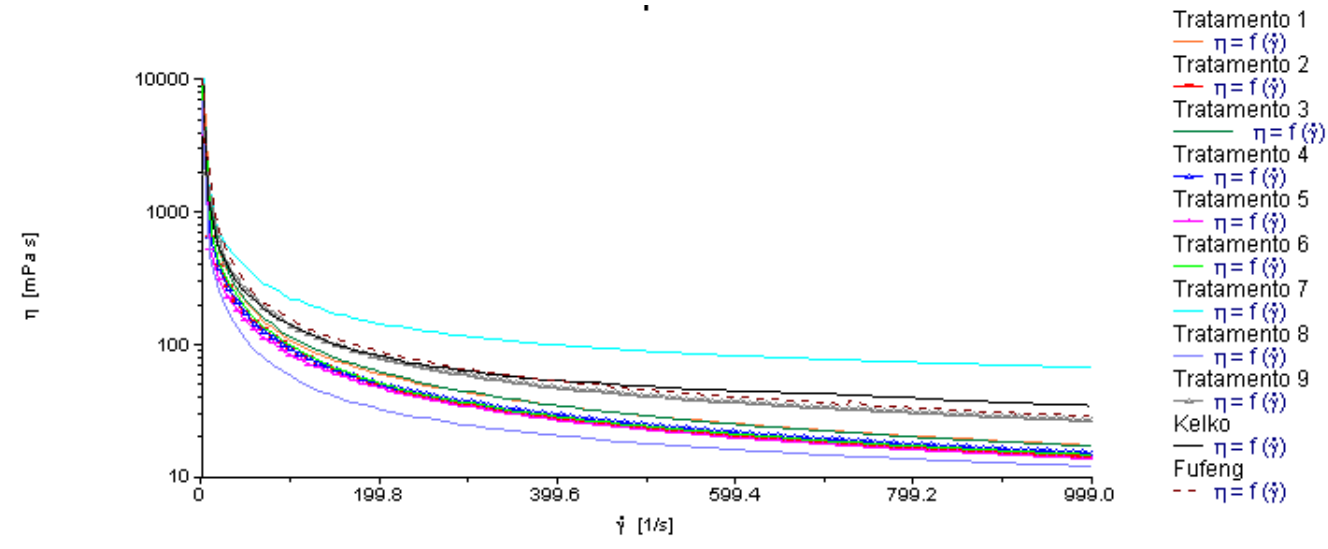

Figura 1 - Viscosidade das xantanas pruni $1 \%(\mathrm{~m} / \mathrm{v})$, em diferentes tratamentos, e comerciais.

Para facilitar a visualização das curvas referentes às medidas oscilatórias foram selecionadas algumas curvas para exemplificar o comportamento das amostras de solução de xantana (Figura 2) e a Tabela 2 sumariza os resultados da análise de viscoelasticidade para todas as xantanas. A xantana 8 teve o segundo maior módulo elástico, sendo precedida pela amostra comercial Fufeng®; e o maior módulo viscoso, seguida da amostra comercial Kelco®.

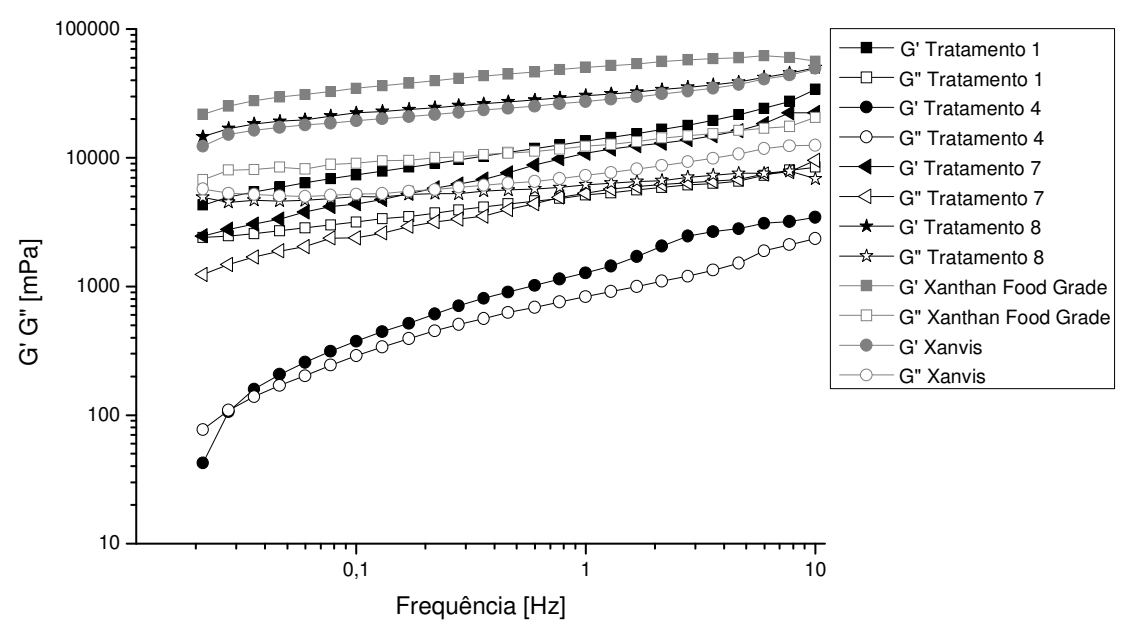

Figura 2 - Resultados das medidas oscilatórias para as xantanas pruni $1 \%(\mathrm{~m} / \mathrm{v})$, em diferentes tratamentos, e comerciais. (Considerar: G' = módulo elástico; G” = módulo viscoso; xanthan food grade $=$ Fufeng ${ }^{\circledR}$ e Xanvis ${ }^{\circledR}=$ Kelco $\left.{ }^{\circledR}\right)$. 


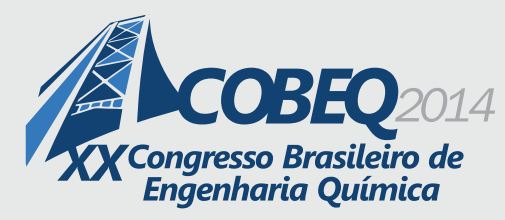

Todas as amostras apresentaram elevação nos módulos elástico e viscoso com o aumento da frequência (Figura 2). Segundo Morris (1992), as soluções de xantana apresentam módulo elástico $\left(\mathrm{G}^{\prime}\right)$ excedendo o módulo viscoso $\left(\mathrm{G}^{\prime \prime}\right)$ em frequências mais elevadas; ou seja, a xantana pode apresentar propriedades similares a gel, o que a confere capacidade de manter partículas em suspensão ou estabilizar emulsões por longos períodos.

Tabela 2 - Medidas oscilatórias e parâmetros reológicos para as soluções de xantanas pruni 1\% $(\mathrm{m} / \mathrm{v})$, em diferentes tratamentos, e comerciais

\begin{tabular}{|c|c|c|c|c|c|c|c|c|c|}
\hline \multirow{4}{*}{ Tratamentos } & \multicolumn{6}{|c|}{ Medidas oscilatórias } & \multirow{3}{*}{\multicolumn{3}{|c|}{ Parâmetros reológicos }} \\
\hline & \multicolumn{6}{|c|}{ Frequência [Hz] } & & & \\
\hline & \multicolumn{2}{|c|}{0,1} & \multicolumn{2}{|c|}{1,0} & \multicolumn{2}{|c|}{10,0} & & & \\
\hline & $\mathrm{G}^{\prime}$ & G” & $\mathrm{G}^{\prime}$ & G” & G' & G” & $\mathrm{K}\left(\mathrm{Pa} \cdot \mathrm{s}^{\mathrm{n}}\right)$ & $\mathrm{n}$ & $\mathrm{R}$ \\
\hline 1 & 7380 & 3160 & 13500 & 5140 & 34100 & 8460 & 2,472 & 0,4544 & 0,9984 \\
\hline 2 & 6940 & 4170 & 14800 & 7100 & 36700 & 9950 & 1,348 & 0,4545 & 0,9995 \\
\hline 3 & 13000 & 4110 & 20700 & 5590 & 18400 & 5070 & 2,086 & 0,4600 & 0,9995 \\
\hline 4 & 375 & 290 & 1270 & 832 & 3450 & 2350 & 1,407 & 0,4501 & 0,9995 \\
\hline 5 & 6550 & 3330 & 12100 & 5020 & 17900 & 7560 & 1,173 & 0,4543 & 0,9995 \\
\hline 6 & 3060 & 2090 & 7900 & 4100 & 20500 & 6250 & 1,576 & 0,4370 & 0,9997 \\
\hline 7 & 4370 & 2390 & 10800 & 5300 & 22400 & 9570 & 3,403 & 0,4660 & 0,9999 \\
\hline 8 & 22300 & 4970 & 30400 & 6160 & 50200 & 6850 & 1,125 & 0,4115 & 0,9997 \\
\hline 9 & 11600 & 4080 & 18400 & 5570 & 27700 & 7800 & 2,837 & 0,4475 & 0,9997 \\
\hline Fufeng ${ }^{\circledR}$ & 34600 & 9070 & 50400 & 12300 & 56400 & 20600 & 2,920 & 0,3775 & 0,9999 \\
\hline Kelco ${ }^{\circledR}$ & 19400 & 5230 & 27500 & 7320 & 49600 & 12500 & 3,002 & 0,4002 & 0,9998 \\
\hline
\end{tabular}

G' = módulo elástico, G” = módulo viscoso, $\mathrm{K}$ = índice de consistência, $\mathrm{n}$ = índice de fluxo, $\mathrm{R}=$ coeficiente de correlação

$\mathrm{O}$ índice de consistência $k$ indica o grau de resistência do fluido diante do escoamento; quanto maior este valor mais viscoso será o fluido. A xantana pruni do tratamento 7 teve maior $\mathrm{k}$, superando as xantanas comerciais. O parâmetro k é de fundamental importância quando se deseja utilizar xantana como agente suspensivo em fluidos de perfuração, para manter em suspensão os cascalhos gerados durante a perfuração. $\mathrm{O}$ índice de fluxo $(n)$ caracteriza o comportamento nãoNewtoniano de um fluido; quanto menor o $\mathrm{n}$ em relação a 1, maior será a pseudoplasticidade, sendo, portanto, mais indicados para emprego na perfuração petrolífera. Em todos os ensaios verificou-se tal comportamento; sendo as mais e menos pseudoplásticas, as xantanas 8 e 7 , respectivamente. O coeficiente de correlação (R), para todos os ensaios, foi superior a 0,99 , indicando uma forte correlação entre os parâmetros (Tabela 2).

No presente trabalho, observou-se uma relação entre as viscosidades (Figura 1) das xantanas e o teor de piruvato (Figura 3a) do polímero; as soluções de maiores viscosidades foram as com maior conteúdo de piruvato (7 e 9); as xantanas que apresentaram uma menor porcentagem deste substituinte foram as de menor viscosidade (8). A literatura preconiza como 1,5\% como valor mínimo de piruvato (Burdock, 1997); entretanto, verificou-se valores menores nas xantanas pruni deste trabalho. Isto demonstra que, apesar do teor de piruvato ser importante, não é principal fator a influenciar na viscosidade. Baixos conteúdos deste constituinte também 


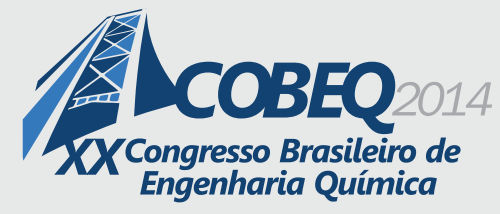

foram relatados por Borges et al. (2009). O acetil (Figura 3b) não mostrou relação direta com a viscosidade. Casas et al. (2000), verificaram que o acetil aumentou com o aumento da agitação, e que não mostrou exercer influência sobre o piruvato. No presente trabalho verificou-se que o piruvato aumentou com a redução da aeração, não mostrando ser influenciado pela agitação; enquanto que os teores de acetil elevaram-se com o aumento da agitação.
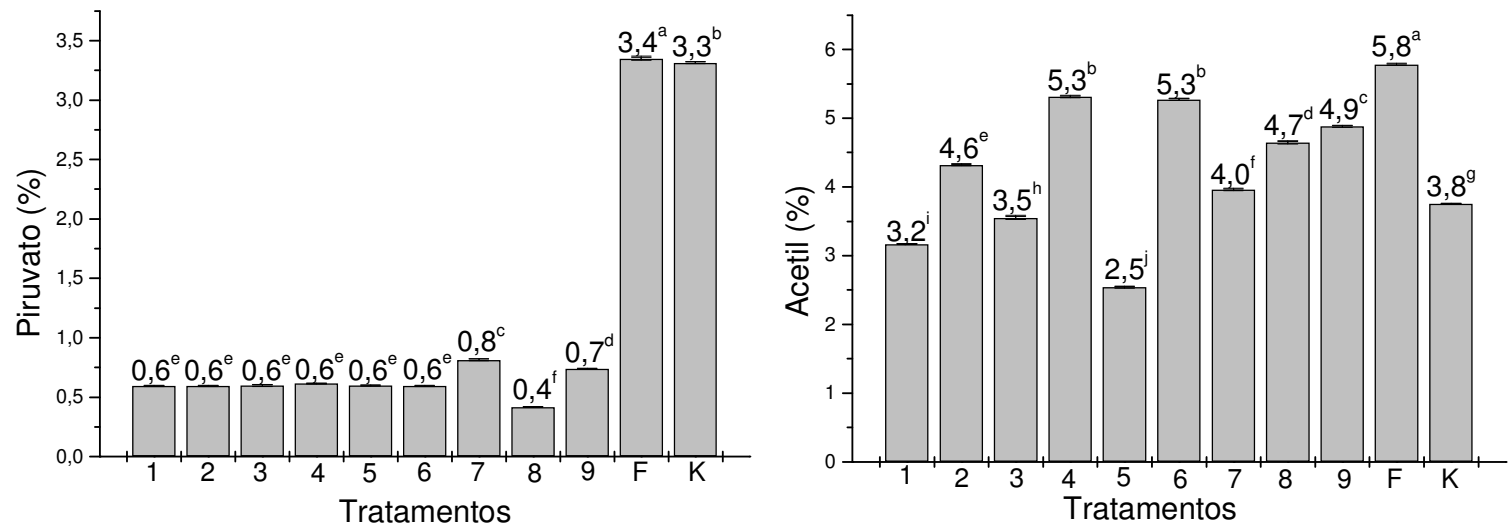

Figura 3 - Teor de (a) piruvato e (b) nas xantanas pruni, em diferentes tratamentos, e comerciais

(F e K). Considerar: letras distintas diferem estatisticamente pelo teste de Tukey com $\mathrm{p}<0,05$; $\mathrm{F}=$ Fufeng® e $\mathrm{K}=$ Kelco ${ }^{\circledR}$.

De acordo com Shatwell et al. (1990), teores de piruvato e acetil (Figura 3a e 3b), respectivamente, de até 8 e 5\%, são teoricamente possíveis; podendo, ainda, haver uma porcentagem maior de grupos acetil. Os baixos valores de piruvato podem ser decorrentes do tratamento térmico do caldo fermentado conduzido em autoclave, como relatado por Borges et al. (2009); sendo este preconizado para inativação celular e enzimática e melhoria das propriedades reológicas da xantana. Embora a caracterização das xantanas não tenha sido analisada em condições salinas, os valores foram similares aos obtidos por Oliveira et al. (2012) para xantana do mesmo patovar; demonstrando potencial como viscosificante em fluidos que utilizam água salina. Há interesse por polímeros com baixo piruvato na recuperação terciária de petróleo. Isso porque apresentam menor adsorção ao solo, com consequente melhora na injetabilidade (Kleinitz et al., 1989).

Tem sido relatada na literatura a ocorrência de outros monossacarídeos na estrutura química da xantana, além da glicose, manose e ácido glicurônico. Moreira et al. (2001) identificaram ramnose na xantana produzida por $X$. arboricola pv pruni, indicando que a espécie influencia na estrutura. A variação dos parâmetros operacionais não influenciou qualitativamente a composição das xantanas pruni; apenas quantitativas, a julgar pelas diferenças entre tamanho e intensidade das manchas (Figura 4). Todas as amostras, assim como as comerciais, apresentaram glicose, ácido glicurônico e manose; ramnose somente foi verificada nas xantanas pruni (1 a 9), como já esperado para a espécie $X$. arboricola. Visualmente, 1, 3, 8 e 9 apresentaram elevada concentração de glicose, demonstrando que a agitação baixa a intermediária influenciou 
positivamente o conteúdo deste. Por outro lado, a agitação elevada a mais elevada influenciou negativamente a concentração de glicose e contribuiu com maior quantidade de manose (4 e 6). Maior teor de manose pode estar relacionado a maiores viscosidade e pseudoplasticidade (Moreira et al., 2001). O emprego de faixa mais ampla de agitação (intermediária a muito elevada) como de aeração (muito baixa a intermediária) ocasionou maiores concentrações de ramnose nos tratamentos 3, 6, 7 e 9, que estão relacionadas ao caráter de gel. Com relação ao ácido gicurônico, foi observado que a agitação intermediária influenciou positivamente este constituinte, que contribui com a solubilidade da molécula. As xantanas comerciais apresentaram as menores concentrações de glicose, manose e ácido glicurônico.

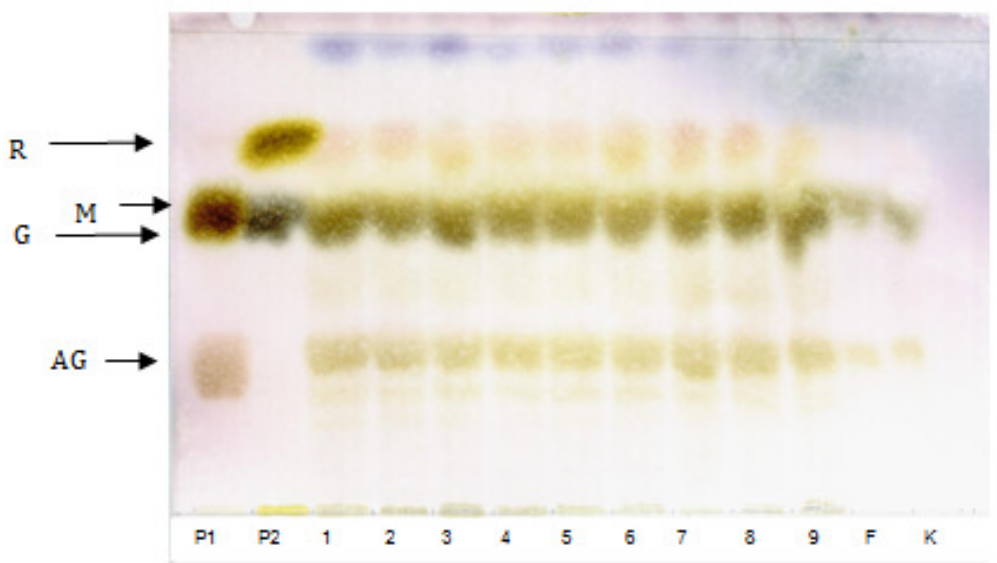

Figura 4 - Cromatografia de camada delgada comparativa de xantanas puni (amostras 1-9) e comerciais ( $F=$ Fufeng $\AA$; $K=$ Kelco $\left.{ }^{\circledR}\right)$; Padrões: $P 1$ - ácido glicurônico $\left(A G, R_{f} 0,30\right)$, manose $(M$, $\left.R_{f} 0,56\right) ; P 2$ - glicose $\left(G, R_{f} 0,54\right)$, ramnose $\left(R, R_{f} 0,69\right)$.

\section{CONCLUSÃO}

A variação da agitação e da aeração influenciaram as propriedades da xantana pruni. A xantana pruni obtido pelo tratamento 7 possui todas as características necessárias a um viscosificante de alta performance para uso em fluido de perfuração de poços de petróleo base água, sendo ainda ambientalmente correto e biocompatível com o ambiente de exploração de petróleo.

\section{REFERÊNCIAS}

BORGES, C. D.; VENDRUSCOLO, C. T.; MARTINS, A. L.; LOMBA, R. F. T. Comportamento reológico de xantana produzida por Xanthomonas arboricola pv pruni para aplicação em fluido de perfuração de poços de petróleo. Polím.: Ciênc. Tecnol., v. 2, 160-165, 2009.

BURDOCK, G. A. Encyclopedia of Food and Color Additives. 3.ed. New York: CRC Press, 1997. 


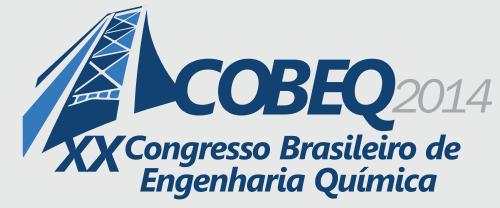

CASAS, J. A.; SANTOS, V. E.; GARCÍA-OCHOA, F. Xanthan gum production under several operational conditions: Molecular structure and rheological properties. Enz. Microbiol. Technol., v. 26, p. 282-291, 2000.

FARIAS, K. V.; AMORIM, L. V.; LIRA, H. L. Desenvolvimento de fluidos aquosos para aplicação em perfuração de poços de petróleo - Parte I, Rer. Eletrôn. Mat. Proc., v.4.1, 2009.

KLEINITZ, W.; LITTMANN, W.; HERBST, H. Screening of xanthan-biopolymer for a high salinity oil reservoir. In: European Symposium on Improved Oil Recovery. Proceedings. Budapest, Hungary, p. 1-9, 1989.

McCOMB, E. A.; McCREADY, R. M. Determination of acetyl in pectin and in acetylated carbohydrate polymers. An. Chem., v. 29, p. 819-821, 1957.

MOREIRA, A. S.; VENDRUSCOLO, J. L. S.; GIL-TUNES, C.; VENDRUSCOLO, C. T. Screening among 18 novel strains of Xanthomonas campestris pv pruni. Food Hyd., v. 15, p. 469-474, 2001.

MOREIRA, A. S.; SOUZA, A. S.; VENDRUSCOLO, C. T. Determinação de composição de biopolímero por cromatografia em camada delgada. Rev. Bras. Agroc., v. 4, p. 222-224, 1998.

MORRIS, V. J. Science, structure and applications of microbial polysaccharides. In: Phillips, G.O.; Wedlock, D.J.; Williams, P.A. Gums and Stabilisers for the Food Industry. Oxford: Pergamon Press, p.315, 1992.

OLIVEIRA, P. D.; BORGES, C. D.; MICHEL, R. C.; LOMBA, R. F. T. ; VENDRUSCOLO, C. T. . Avaliação Comparativa das Propriedades de Xantanas Produzidas pelo patovar pruni e Clairana com Xantana Comercial para predição de uso. Polím. (São Carlos. Online), 2012 (in press).

SHATWELL, K. P.; SUTHERLAND, I. W.; DEA, I. C.M.; ROSS-MURPHY, S. B. The influence of acetyl and pyruvate substituents on the helix-coil transition behavior of xanthan. Carb. Res., v. 206, p. 87-103, 1990.

SLONEKER, J. H.; JEANES, A. Exocellular bacterial polysaccharide from Xanthomonas campestris NRRL B - 1459. Can. J. Chem., Ottawa, v. 40, n. 11, p. 2066-2071, 1962.

TORRES, L. G.; BRITO, E.; GALINDO, E.; CHOPLIN, L. Viscous behavior of xanthan solutions from a variant strain of Xanthomonas campestris. J. Ferm. Bioeng., v. 75, 58-64, 1993.

UNIVERSIDADE FEDERAL DE PELOTAS. VENDRUSCOLO; C. T.; VENDRUSCOLO; J. L. S.; MOREIRA, A. S. Process for preparing a xanthan biopolymer.WO/2006/047845. 2006.

VENDRUSCOLO, C. T.; MOREIRA, A. S.; SOUZA, A. S.; ZAMBIAZI, R.; SCAMPARINI, A. R. P. Heteropolysaccharide produced by Xanthomonas campestris pv pruni C24. In: NISHINARI, K. Hydrocolloids. Amsterdam: Elsevier, v. 1, p. 187-191, 2000.

XUEWU, Z.; XIN, L.; DEXIANG, G.; WEI, Z.; TONG, X.; YONGHONG, M. Rheological models for xanthan gum. J. Food Eng., v. 27, p. 203-209, 1996. 